\title{
WORKFORCE Employment of medical and physician associate students in an NHS trust during the COVID-19 pandemic
}

\author{
Authors: Katie Maas, ${ }^{A}$ Marian Wassef, ${ }^{B}$ Milind Kulkarni ${ }^{C}$ and Lesley Bowker ${ }^{D}$
}

\begin{abstract}
We present a model of employment of healthcare professional students successfully used during the COVID-19 pandemic to support and increase the local workforce. Following recruitment, students from multiple year groups, with varying experience, were deployed to many areas within the trust. The model used allowed overseeing staff to re-deploy students as required in response to changing demand. We received positive feedback from staff and students throughout and present the analysis of a student survey performed towards the end of their roles. We hope the model provides vital insight and an example for other trusts should future need arise during the COVID-19 pandemic and beyond.
\end{abstract}

KEYWORDS: employment, pandemic, COVID-19, medical student, physician associate student

DOI: $10.7861 /$ fhj.2020-0174

\section{Introduction}

We present a model utilised at the Norfolk and Norwich University Hospital (NNUH) during the COVID-19 pandemic to enrol medical and physician associate students to work within our NHS tertiary trust. Following cancellation of clinical placements in medical schools across the country and increasing demand within the NHS to expand the workforce during the pandemic, the trust responded by creating the roles of senior and junior student clinical assistants (SCAs). ${ }^{1-3}$

We were overwhelmed with the willingness to help from students. This response was mirrored nationwide, with many healthcare students expressing desire to help their colleagues and future employers. ${ }^{4,5}$ In addition, many students had understandably expressed concern with the resulting lack of clinical exposure from cancelled placements and this role provided a partial solution to this apprehension. ${ }^{4}$

Authors: ${ }^{\text {A ST1 }}$ obstetrics and gynaecology, Ipswich Hospital, Ipswich, UK; ${ }^{B}$ CT1 anaesthetics, Scarborough General Hospital, Scarborough, UK; ${ }^{C}$ consultant in paediatric and neonatal surgery and associate medical director, Norfolk and Norwich University Hospital, Norwich, UK; ${ }^{D}$ clinical skills director, Norwich Medical School, Norwich, UK and consultant in older people's medicine, Norfolk and Norwich University Hospital, Norwich, UK
We were able to recruit students in liaison with Norwich Medical School (NMS) with strong support from the trust's medical director and medical staffing department. Students who volunteered were employed on bank agreements to allow flexibility with working hours and to tailor the level of support provided to the trust.

The model was unusual in its scale and speed of implementation with students working from 20 March 2020 (Fig 1). We employed over 250 students before the end of April 2020, with the majority deployed prior to the peak of the pandemic and prior to introduction of the foundation interim year-1 (FiY1) role. ${ }^{6}$

\section{Methodology}

We initially enlisted year-5 medical students who were assigned to specialty teams. The number of students allocated to each team was determined by clinical need. This allowed departments to support rota gaps resulting from the pandemic, compensating for staff required to isolate or shield.

After this deployment, we invited year-4 medical students, year-2 physician associate students and medical students who lived locally but were studying at medical schools other than NMS. Later, local year-1-3 students were invited to avoid unnecessary travel to the area.

We designated year- 4 and 5 students as senior SCAs, with payment agreed at 'band 4', and year-1-3 students as junior SCAs, with 'band 3' payment. The role involved supporting medical teams with tasks suited to individual competency to reflect their level of training and to ensure they were covered by trust indemnity. Responsibilities included clerking patients, assisting on ward rounds, performing practical skills, writing discharge summaries and communicating with patient's next of kin, among other tasks. They did not have prescribing privileges or the ability to order ionising radiation.

We organised training and recruitment over 7 days, employing a total of 259 students to work within the trust. Recruitment events included medical staffing agreements, setting up IT accounts, COVID-19 risk assessment, a briefing talk as well as demonstrations on donning and doffing, and practical skills refreshers. Social distancing was ensured by staggering start times and maintaining bubbles of 10 students throughout the process. We provided testing for FFP3 masks prior to commencing work and ensured that they knew how to access support. Students were able to start immediately after recruitment as employment checks were completed in parallel with commencement of posts. This minor risk was seen as justifiable as all local students had already been cleared for trust clinical placements. 


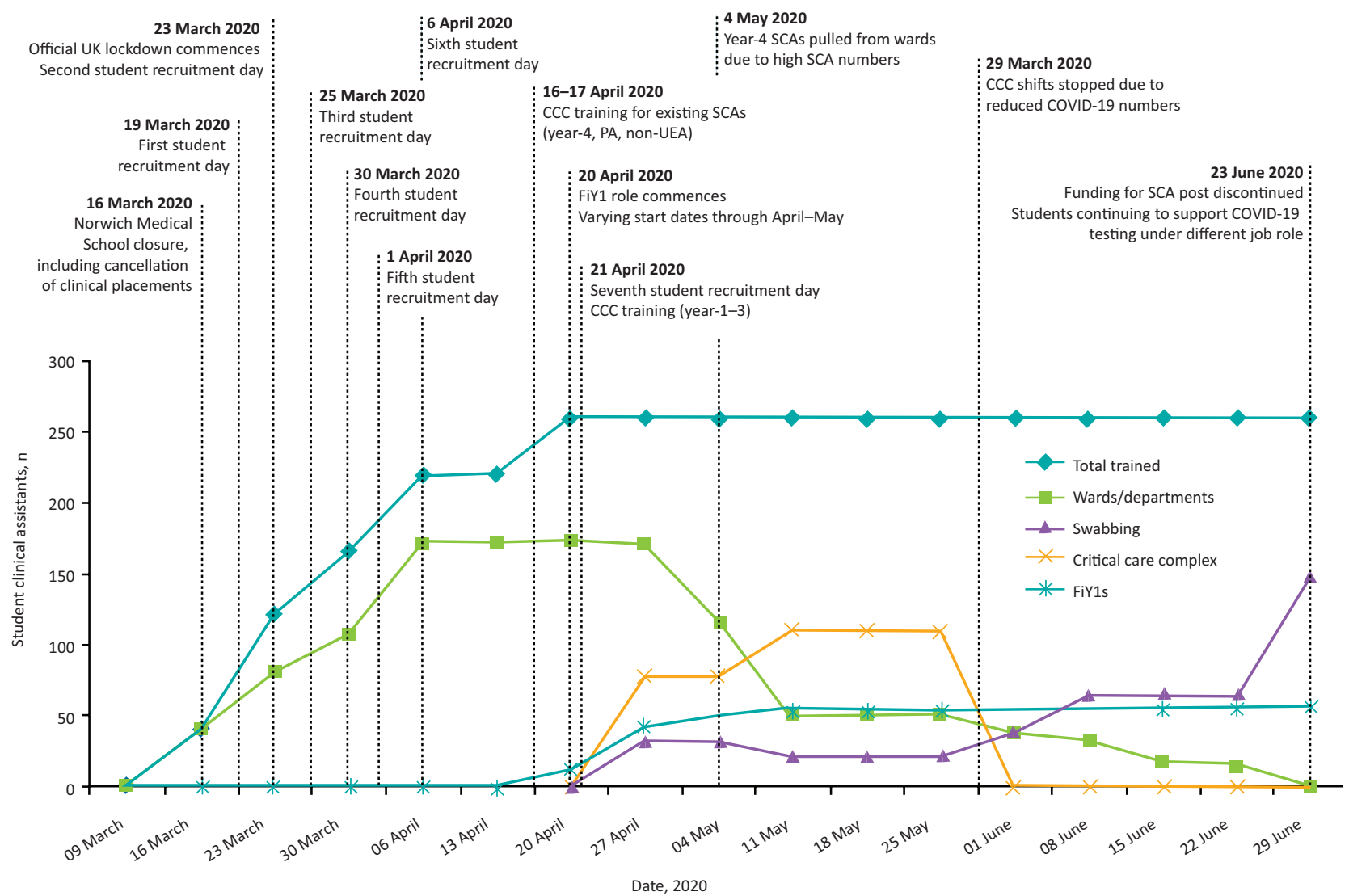

Fig 1. Recruitment timeline of student clinical assistants. $C C C=$ critical care complex; FiY1 = foundation interim year-1 doctor; PA = physician associate; SCA = student clinical assistant; UEA = University of East Anglia.

As the pandemic evolved, our critical care complex (CCC) was designated a 'surge hospital' for local trusts. In response, we trained year-1-4 SCAs to undertake a healthcare assistant role in CCC assisting with tasks such as basic nursing care and proning. The SCAs also supported COVID-19 swab testing which commenced at the trust following government initiatives; a role for which other institutions also recruited students. ${ }^{7}$ Initially year-4 SCAs also worked alongside year-5 SCAs but as numbers of students on wards increased, we responded by re-distributing year4 SCAs to CCC and COVID-19 testing roles only.

Our team oversaw all students, including shift booking for year-1-4 SCAs. This allowed us to provide pastoral care, ensure student safety and wellbeing, and encourage students to balance university commitments once online lecture programmes recommenced.

\section{Outcome}

Throughout the student deployment period, we were able to continually monitor the impact of SCAs on clinical teams. We had regular contact with students, as well as departmental leads. We received only positive feedback from colleagues, commending students on their clinical and interprofessional skills by integrating well into teams.

A small number of students $(n=2)$ were risk assessed as unsuitable to work in areas with COVID-19 present on occupational or social grounds. Throughout employment, only one student was confirmed to have contracted COVID-19, although this was only after testing had commenced. Several others were unable to work for short periods of time, as they were required to self-isolate. This was largely due to contact with cases in their household, but also presence of symptoms themselves.

With the introduction of the FiY1 role in May 2020, 52.8\% of year- 5 SCAs transitioned into this new position. ${ }^{6}$ Others opted to continue in SCAs roles; this was for a variety of reasons including high FiY1 application rate for our trust, inability to re-locate to other areas easily, feeling integrated in clinical teams and the flexibility of the bank working pattern. Of the remaining year-5 SCAs, most terminated their roles towards the end of June 2020, prior to commencing foundation year-1 (FY1) training in August 2020. As year-5 SCAs exited the role, they were sent a letter of appreciation with certificate and survey invitation. The survey was also adapted and sent to year-4 SCAs in May 2020 to capture views of students continuing to work. The response rate was $67.7 \%$ for year- 4 and $53.0 \%$ for year- 5 SCAs.

The analysis was similar for both year groups, despite their varied working environments and study commitments (Fig 2). They worked a variety of shifts, including antisocial hours at their discretion. Most students felt well supported by clinical teams (96.6\%), did not feel pressured to perform tasks outside of their capability (89.9\%) and were satisfied by the pay arrangement 


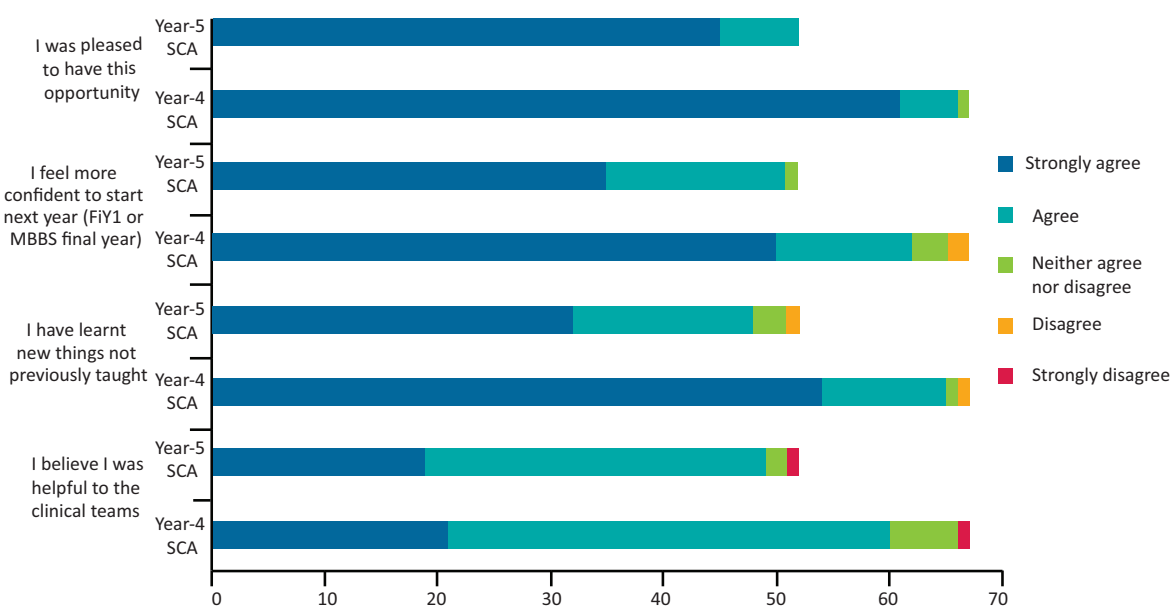

Fig 2. Survey results of year-4 $(n=67)$ and year-5 $(n=52)$ student clinical assistants. FiY1 = foundation interim year1 doctor; SCA = student clinical assistant
(87.4\%). Interestingly, many stated they had volunteered not expecting payment, but appreciated financial support. Additionally, year- 4 SCAs overall felt they were able to balance the role with university commitments and asked for this opportunity to be extended to other year groups.

The biggest obstacle faced during this process was initiating payment; predominantly due to our recruitment team being overwhelmed with unplanned new recruits. While some students waited up to 3-weeks for their initial payment, there was an immense support from medical staffing and payroll staff, and all students were paid weekly by the end of the programme.

As COVID-19 numbers in the area continued to reduce, funding for the role ceased from 29 June 2020. Prior to this, CCC shifts had already stopped and ward deployment reduced. Students assisting with COVID-19 testing continued to work under different titles as the need for swabbing continued to increase with the reintroduction of elective inpatients.

\section{Conclusion and next steps}

The creation of the senior and junior SCA role has played a key part in the COVID-19 response within our trust. Our success was due to our team quickly identifying and implementing a role which would benefit both students and clinical teams. This was achieved with support from the trust's directors, allowing deployment before the height of the pandemic.

Another reason for success was the use of a dedicated team of administrative and medical staff. The team were already employed within undergraduate medical education at the trust, whose usual roles were to oversee students' medical placements. This was vital in providing ongoing support and pastoral care; although many students showed eagerness to help, there was also inevitable uncertainty.

The team also continually monitored student allocations and responded to feedback to ensure SCAs were placed in areas of greatest clinical need. As COVID-19 numbers decreased, the need for support was continually re-evaluated; some SCA roles ceased and students were redistributed. This flexibility allowed us to easily oversee 'sign-up' of shifts, permitting students to work when it suited them.

The overwhelmingly positive response from clinical staff and students alike raises the possibility of future considerations to introduce similar voluntary roles nationwide, irrespective of the pandemic, aimed at flexibly supporting NHS staffing while benefiting students' learning and financial position.

If such a model is required again, an area of suggested review is exploring the use of virtual training. Although we feel many aspects of training should be face-to-face (ie practical skills), some aspects (such as administration) could easily be undertaken virtually. This would reduce infection transmission risk, especially with the improved infrastructure for virtual training secondary to the pandemic.

Overall, our experience of healthcare professional student recruitment to increase the trust's workforce during the COVID-19 pandemic has been positive and something which can be adapted if future need arises, especially in event of another peak. We have presented a model focused on combining the flexibility of bank agreements and an overseeing team which, combined with our students' enthusiasm and professionalism, we feel has contributed most significantly to the role's success.

\section{Acknowledgements}

The authors wish to acknowledge the administrative and clinical staff from the Clinical Skills Resource Area, Norfolk and Norwich Hospital, as well as Norwich Medical School executive staff for their support; without this, our model would not have been possible. We also wish to acknowledge Norfolk and Norwich Hospital medical director for their early support and encouragement.

\section{References}

1 Mahase E. COVID-19: medical students to be employed by NHS. BMJ 2020;368:m1156.

2 Representatives of STARSurg Collaborative, EuroSurg Collaborative, TASMAN Collaborative. Medical student involvement in the COVID-19 response. Lancet 2020;395:1254.

3 Rasmussen S, Sperling P, Poulsen M, Emmersen J, Andersen S. Medical students for health-care staff shortages during the COVID-19 pandemic. Lancet 2020;395:e79-80. 
4 Thomson E, Lovegrove S. 'Let us Help' - Why senior medical students are the next step in battling the COVID-19 pandemic. Int J Clin Pract 2020;74:e13516.

5 Miller D, Pierson L, Doernberg S. The role of medical students during the COVID-19 pandemic. Ann Intern Med 2020;173:145-6.

6 Harvey A. COVID-19: medical students and FY1 doctors to be given early registration to help combat COVID-19. BM] 2020;368:m1268.
7 Klasen J. SWAB team instead of SWAT team - students as frontline force during the COVID-19 pandemic. Med Educ 2020;54:860

Address for correspondence: Dr Katie Maas, Obstetrics and Gynaecology Department, Ipswich Hospital, Heath Road, Ipswich IP4 5PD, UK.

Email:katie.maas@doctors.org.uk

Twitter: @KatieMarieMaas 